\title{
$S U(3)_{\mathrm{L}} \rtimes\left(\mathbb{Z}_{3} \times \mathbb{Z}_{3}\right)$ gauge symmetry and Tri-bimaximal mixing
}

\author{
Chuichiro HATTORI, * Mamoru MATSUNAGA, ${ }^{a}+$ \\ Takeo MATSUOKA ${ }^{a} \ddagger$ and Kenichi NAKANISHI, ${ }^{b}$ § \\ Science Division, General Education, Aichi Institute of Technology, \\ Toyota 470-0392, JAPAN \\ ${ }^{a}$ Department of Physics Engineering, Mie University, \\ Tsu 514-850\%, JAPAN \\ ${ }^{b}$ Department of Sustainable Resource Science, Mie University, \\ Tsu 514-8507, JAPAN
}

\begin{abstract}
We study an effective gauge theory whose gauge group is a semidirect product $G=G_{c} \rtimes \Gamma$ with $G_{c}$ and $\Gamma$ being a connected Lie group and a finite group, respectively. The semidirect product is defined through a projective homomorphism $\gamma$ (i.e., homomorphism up to the center of $G_{c}$ ) from $\Gamma$ into $G_{c}$. The (linear) representation of $G$ is made from $\gamma$ and a projective representation of $\Gamma$ over $\mathbb{C}$. To be specific, we take $S U(3)_{L}$ as $G_{c}$ and $\mathbb{Z}_{3} \times \mathbb{Z}_{3}$ as $\Gamma$. It is noticed that the irreducible projective representations of $\Gamma$ are three-dimensional in spite of its Abelian nature. We give a toy model on the lepton mixing which illustrates the peculiar feature of such gauge symmetry. It is shown that under a particular vacuum alignment the tri-bimaximal mixing matrix is reproduced.
\end{abstract}

\footnotetext{
*E-mail: hattori@aitech.ac.jp

${ }^{\dagger}$ E-mail: matsuna@phen.mie-u.ac.jp

${ }^{\ddagger}$ E-mail: t-matsu@siren.ocn.ne.jp

$\S$ deceased
} 


\section{Introduction}

Current data on lepton mixing show that the mixing matrix takes a peculiar form which is approximately tri-bimaximal.[1] In order to unify the three families into irreducible flavor triplets and to reproduce the specific mixing pattern, many authors have searched for various types of non-Abelian flavor symmetry. 2] A large number of flavor models on lepton mixing have been constructed. In these attempts the authors have taken up a direct product of the flavor group $\Gamma$ with the connected gauge group $G_{c}$. However, it is possible to construct models not only with direct product groups but also with semidirect product groups. We will focus our attention in this paper on a semidirect product gauge group $G_{c} \rtimes \Gamma$.

It has been argued that all non-gauge symmetries are strongly violated by quantum gravity effects around the Planck scale and hence in the low-energy effective theory we cannot have any global symmetries including discrete groups. [3] For this reason the flavor group $\Gamma$ which appears in the low-energy effective theory should be a gauge symmetry. Consequently, as for the choice of the gauge symmetry it is legitimate to adopt the semidirect product group $G_{c} \rtimes \Gamma$.

In the case of the direct product gauge symmetry $G_{c} \times \Gamma$, if the flavor group $\Gamma$ is Abelian, it is impossible to have irreducible flavor triplets. However, in irreducible linear representations of the semidirect product group $G=G_{c} \rtimes \Gamma$, even if $\Gamma$ is Abelian, it is possible to have flavor triplets which are irreducible projective representations of $\Gamma$. In view of this, we study in this paper the effective theory with the semidirect product gauge symmetry $G=G_{c} \rtimes \Gamma=S U(3)_{\mathrm{L}} \rtimes\left(\mathbb{Z}_{3} \times \mathbb{Z}_{3}\right)$. As an illustrative example, we give a toy model on the lepton mixing in the context of the supersymmetric effective theory with this gauge symmetry. In this model three families of leptons are assigned into flavor triplets of the projective representation of $\Gamma=\mathbb{Z}_{3} \times \mathbb{Z}_{3}$.

This paper is organized as follows. In section 2 we give a brief explanation of the semidirect product group $G=G_{c} \rtimes \Gamma$ and its irreducible representations. Our emphasis is placed on the projective homomorphism (i.e., homomorphism up to the center of $G_{c}$ ) of $\Gamma=\mathbb{Z}_{3} \times \mathbb{Z}_{3}$ into $G_{c}=S U(3)$. In section 3 we present a toy model which illustrates the peculiar features of the semidirect product gauge group. We show that under a particular vacuum alignment the tri-bimaximal lepton mixing can be reproduced. Final section is devoted to summary. 


\section{Semidirect product group and its representa- tions}

The semidirect product group $G=G_{c} \rtimes \Gamma$ which we consider in this paper has the multiplication rule

$$
(g, s)\left(g^{\prime}, s^{\prime}\right)=\left(g \imath_{s}\left(g^{\prime}\right), s s^{\prime}\right)
$$

for $(g, s),\left(g^{\prime}, s^{\prime}\right)$ with $g, g^{\prime} \in G_{c}$ and $s, s^{\prime} \in \Gamma$. The symbol $\imath$ represents a homomorphism from $\Gamma$ to the inner automorphism group of $G_{c}$

$$
\begin{aligned}
\imath: \Gamma & \longrightarrow \operatorname{Inn}\left(G_{c}\right) \\
s & \longmapsto \imath_{s}(\bullet)=\gamma_{s}(\bullet) \gamma_{s}^{-1}
\end{aligned}
$$

with $\gamma_{s}$ being an element of $G_{c}$.

The homomorphic nature of $\imath$ requires that the product of $\gamma_{s}$ and $\gamma_{s^{\prime}}$ is equal to $\gamma_{s s^{\prime}}$ up to the center of $G_{c}$, i.e.,

$$
\gamma_{s} \gamma_{s^{\prime}}=f_{s, s^{\prime}} \gamma_{s s^{\prime}} \quad\left(f_{s, s^{\prime}} \in Z\left(G_{c}\right)\right)
$$

which means that the map

$$
\begin{aligned}
\gamma: \Gamma & \longrightarrow G_{c} \\
s & \longmapsto \gamma_{s} .
\end{aligned}
$$

is a homomorphism up to the center, hence called in this paper a projective homomorphism. The set $\left\{f_{s, s^{\prime}}\right\}_{s, s^{\prime} \in \Gamma}$ is called a factor set associated with $\gamma$. The associative law of the products among $\gamma_{s}$ 's leads to the so-called cocycle condition on the factor set. By multiplying $\gamma_{s}$ by an element of $Z\left(G_{c}\right)$, we may redefine $\gamma_{s}$. If all elements of the factor set are reducible to unity through this redefinition, it can be shown that the semidirect group is isomorphic to the direct product group. [4] In other words, in the genuine projective homomorphism we can not reduce all of $f_{s, s^{\prime}}$ 's to unity via such redefinition.

We now take $G_{c}=S U(3)$ and

$$
\Gamma=\mathbb{Z}_{3} \times \mathbb{Z}_{3}=\left\langle a, b \mid a^{3}=b^{3}=e, b a=a b\right\rangle .
$$


For the projective homomorphism from $\Gamma=\mathbb{Z}_{3} \times \mathbb{Z}_{3}$ to $S U(3)$, we can set, through the redefinition of $\gamma_{s}$,

$$
\begin{aligned}
\gamma_{a}^{3} & =f_{a} \mathbf{1}, \\
\gamma_{b}{ }^{3} & =f_{b} \mathbf{1}, \\
\gamma_{b} \gamma_{a} & =f_{c} \gamma_{a} \gamma_{b}
\end{aligned}
$$

with $f_{a, b, c} \mathbf{1} \in Z(S U(3))$. [4] In the following we choose $f_{a}=f_{b}=1$ and $f_{c}=\omega=$ $\exp (2 \pi i / 3)$.

An irreducible linear representation of $G=S U(3) \rtimes \Gamma$ is expressed as

$$
\mathcal{R}(g, s)=R\left(g \gamma_{s}\right) \otimes \rho_{s}^{*}
$$

where $R(g)$ is a representation of $g \in S U(3)$ and $\rho_{s}$ stands for a projective representation of $\Gamma$ over $\mathbb{C}$ whose factor set is the same as that of $R\left(\gamma_{s}\right)$. In the product of $\mathcal{R}(g, s)$ and $\mathcal{R}\left(g^{\prime}, s^{\prime}\right)$, the factor sets coming from $R$ and $\rho$ cancell out and hence $\mathcal{R}$ forms a linear representation.

When we take the trivial representation for $R, \rho$ is one of the irreducible linear representation of $\mathbb{Z}_{3} \times \mathbb{Z}_{3}$, which are all singlets distinguished by the characters

$$
\left(\chi_{a}, \chi_{b}\right)=\left(\omega^{k}, \omega^{l}\right)
$$

with $k, l=0, \pm 1$. We denote these singlets by $\mathbf{1}_{(k, l)}$. When we take the fundamental representation 3 (anti-fundamental representation $3^{*}$ ) for $R, \rho$ is the irreducible projective representation $\mathbf{3}\left(\mathbf{3}^{*}\right)$ of $\mathbb{Z}_{3} \times \mathbb{Z}_{3}$, which satisfies [5]

$$
\begin{aligned}
\rho_{a}{ }^{3} & =\mathbf{1}, \\
\rho_{b}{ }^{3} & =\mathbf{1}, \\
\rho_{b} \rho_{a} & =f_{c}^{\prime} \rho_{a} \rho_{b}
\end{aligned}
$$

with $f_{c}^{\prime}=\omega\left(\omega^{*}\right)$. The projective unitary representation $\mathbf{3}$, in a basis where $\rho_{a}$ is diagonal, is given by

$$
\rho_{a}=\left(\begin{array}{ccc}
1 & 0 & 0 \\
0 & \omega & 0 \\
0 & 0 & \omega^{2}
\end{array}\right), \quad \rho_{b}=\left(\begin{array}{ccc}
0 & 0 & 1 \\
1 & 0 & 0 \\
0 & 1 & 0
\end{array}\right)
$$


In this projective representation of $\Gamma$ the multiplication rules are

$$
\begin{aligned}
& 3 \times 3=3^{*}+3^{*^{\prime}}+3^{*^{\prime \prime}} \\
& 3 \times 3^{*}=\sum_{k, l=0, \pm 1} \mathbf{1}_{(k, l)} .
\end{aligned}
$$

The product rules of two triplets $\left(x_{1}, x_{2}, x_{3}\right)$ and $\left(y_{1}, y_{2}, y_{3}\right)$ turn out to be

$$
\left(\begin{array}{l}
x_{1} y_{1} \\
x_{2} y_{2} \\
x_{3} y_{3}
\end{array}\right) \sim \mathbf{3}^{*}, \quad\left(\begin{array}{l}
x_{2} y_{3} \\
x_{3} y_{1} \\
x_{1} y_{2}
\end{array}\right) \sim \mathbf{3}^{*^{\prime}}, \quad\left(\begin{array}{c}
x_{3} y_{2} \\
x_{1} y_{3} \\
x_{2} y_{1}
\end{array}\right) \sim \mathbf{3}^{*^{\prime \prime}}
$$

The product rules of a triplet $\left(x_{1}, x_{2}, x_{3}\right)$ and an anti-triplet $\left(\bar{y}_{1}, \bar{y}_{2}, \bar{y}_{3}\right)$ become

$$
\begin{aligned}
& x_{1} \bar{y}_{1}+x_{2} \bar{y}_{2}+x_{3} \bar{y}_{3} \sim \mathbf{1}_{(0,0)}, \\
& x_{1} \bar{y}_{1}+\omega^{2} x_{2} \bar{y}_{2}+\omega x_{3} \bar{y}_{3} \sim \mathbf{1}_{(0,1)}, \\
& x_{1} \bar{y}_{1}+\omega x_{2} \bar{y}_{2}+\omega^{2} x_{3} \bar{y}_{3} \sim \mathbf{1}_{(0,-1)}, \\
& x_{1} \bar{y}_{3}+x_{2} \bar{y}_{1}+x_{3} \bar{y}_{2} \sim \mathbf{1}_{(1,0)}, \\
& x_{1} \bar{y}_{3}+\omega^{2} x_{2} \bar{y}_{1}+\omega x_{3} \bar{y}_{2} \sim \mathbf{1}_{(1,1)}, \\
& x_{1} \bar{y}_{3}+\omega x_{2} \bar{y}_{1}+\omega^{2} x_{3} \bar{y}_{2} \sim \mathbf{1}_{(1,-1)}, \\
& x_{1} \bar{y}_{2}+x_{2} \bar{y}_{3}+x_{3} \bar{y}_{1} \sim \mathbf{1}_{(-1,0)}, \\
& x_{1} \bar{y}_{2}+\omega^{2} x_{2} \bar{y}_{3}+\omega x_{3} \bar{y}_{1} \sim \mathbf{1}_{(-1,1)}, \\
& x_{1} \bar{y}_{2}+\omega x_{2} \bar{y}_{3}+\omega^{2} x_{3} \bar{y}_{1} \sim \mathbf{1}_{(-1,-1)} .
\end{aligned}
$$

Generally, the linear representations of the semidirect product gauge group $S U(p) \rtimes$ $\left(\mathbb{Z}_{p} \times \mathbb{Z}_{p}\right)$ with a prime number $p$ stand in need of the projective representation of $\Gamma=\mathbb{Z}_{p} \times \mathbb{Z}_{p}$, which is designated by the relation $\rho_{b} \rho_{a}=f_{c}^{\prime} \rho_{a} \rho_{b}$ with $f_{c}^{\prime}=$ $\exp (2 \pi i m / p), m=0,1, . ., p-1$. The irreducible representations are $p^{2}$ singlets for $m=0$ (linear representations) and $p$-plet for each $m \neq 0$ (genuine projective representation).

It is expected that the theory with the semidirect product gauge symmetry contains interesting features different from the one with the direct product gauge symmetry. To see this concretely, taking $G=S U(3)_{L} \rtimes\left(\mathbb{Z}_{3} \times \mathbb{Z}_{3}\right)$ gauge group as an example, we formulate a toy model on the lepton mixing. 


\section{A toy model and tri-bimaximal mixing}

Here we concentrate our attention on the mixing matrix in the lepton sector. It is assumed that the gauge symmetry $S U(2)_{\mathrm{L}}$ is enlarged to $S U(3)_{\mathrm{L}}$ and that $S U(2)_{\mathrm{L}^{-}}$ doublet and -singlet leptons are assigned into $(R, \rho) \sim(\mathbf{3}, \mathbf{3})$ as

$$
\vec{L}=\left(\begin{array}{c}
* \\
\nu_{\mathrm{L}} \\
l_{\mathrm{L}}^{-}
\end{array}\right), \quad \overrightarrow{l^{c}}=\left(\begin{array}{c}
l_{\mathrm{R}}{ }^{c} \\
* \\
*
\end{array}\right) \text {. }
$$

The fields denoted by asterisks do not affect the following discussions. We now adopt a supersymmetric context and introduce two kinds of Higgs fields, which are $(R, \rho) \sim(\mathbf{3}, \mathbf{3})$ and $\left(\mathbf{3}^{*}, \mathbf{3}^{*}\right)$ denoted as

$$
\vec{\phi}=\left(\begin{array}{c}
* \\
\phi^{0} \\
\phi^{-}
\end{array}\right), \quad \vec{\varphi}=\left(\begin{array}{c}
\varphi^{+} \\
\varphi^{0} \\
*
\end{array}\right)
$$

respectively. Incidentally, $\vec{L}$ and $\overrightarrow{l^{c}}$ represent odd R-parity superfields, while $\vec{\phi}$ and $\vec{\varphi}$ even R-parity ones. The product rules among flavor triplets and anti-triplets such as $\left(\overrightarrow{L_{1}}, \overrightarrow{L_{2}}, \overrightarrow{L_{3}}\right),\left(\overrightarrow{l_{1}^{c}}, \overrightarrow{l_{2}^{c}}, \overrightarrow{l_{3}^{c}}\right)$, etc. are given in the previous section.

We next proceed to study the $G$-invariant operators in the superpotential. Taking account of the fact that the element $(g, s) \in G=G_{c} \rtimes \Gamma$ is decomposed as $(g, s)=$ $(g, e)(e, s)$ uniquely, we can replace the $G$-invariant condition on the operators by the separate requirements of the $G_{c}$-invariance and $\Gamma$-invariance. Charged lepton masses arise from the $S U(3)_{\mathrm{L}}$-invariant Yukawa couplings $\vec{L} \overrightarrow{l c} \vec{\phi}$ in the superpotential. The flavor structure of the Yukawa couplings $\vec{L} \overrightarrow{l^{c}} \vec{\phi}$ is of the form

$$
\begin{array}{r}
h_{1}\left(\overrightarrow{L_{1}} \overrightarrow{l_{1}^{c}} \overrightarrow{\phi_{1}}+\overrightarrow{L_{2}} \overrightarrow{l_{2}^{c}} \overrightarrow{\phi_{2}}+\overrightarrow{L_{3}} \overrightarrow{l_{3}^{c}} \overrightarrow{\phi_{3}}\right)+h_{2}\left(\overrightarrow{L_{1}} \overrightarrow{l_{2}^{c}} \overrightarrow{\phi_{3}}+\overrightarrow{L_{2}} \overrightarrow{l_{3}^{c}} \overrightarrow{\phi_{1}}+\overrightarrow{L_{3}} \overrightarrow{l_{1}^{c}} \overrightarrow{\phi_{2}}\right) \\
+h_{3}\left(\overrightarrow{L_{1}} \overrightarrow{l_{3}^{c}} \overrightarrow{\phi_{2}}+\overrightarrow{L_{2}} \overrightarrow{l_{1}^{c}} \overrightarrow{\phi_{3}}+\overrightarrow{L_{3}} \overrightarrow{l_{2}^{c}} \overrightarrow{\phi_{1}}\right) .
\end{array}
$$

When the Higgs fields $\phi_{i}^{0}(i=1,2,3)$ acquire their vacuum expectation values $v_{i}$, we 
obtain the charged lepton mass matrix

$$
M_{l}=\left(\begin{array}{lll}
h_{1} v_{1} & h_{2} v_{3} & h_{3} v_{2} \\
h_{3} v_{3} & h_{1} v_{2} & h_{2} v_{1} \\
h_{2} v_{2} & h_{3} v_{1} & h_{1} v_{3}
\end{array}\right)
$$

If we assume the vacuum configuration

$$
v_{1}=v_{2}=v_{3}
$$

the charged lepton mass matrix $M_{l}$ is diagonalized through the unitary transformation $U_{l}^{\dagger} M_{l} U_{l}$. The unitary matrix $U_{l}$ becomes

$$
U_{l}=\frac{1}{\sqrt{3}}\left(\begin{array}{ccc}
1 & 1 & 1 \\
1 & \omega & \omega^{2} \\
1 & \omega^{2} & \omega
\end{array}\right)
$$

Light neutrino masses are described by the non-renormalizable superpotential term $\vec{L} \vec{L} \vec{\varphi} \vec{\varphi}$, where $\vec{L}$ and $\vec{\varphi}$ is assigned into $(R, \rho) \sim(\mathbf{3}, \mathbf{3})$ and $\left(\mathbf{3}^{*}, \mathbf{3}^{*}\right)$, respectively. There are two types of the non-renormalizable operators [6]

$$
(\vec{L} \vec{L})_{\mathbf{3}^{*}}(\vec{\varphi} \vec{\varphi})_{\mathbf{3}}, \quad(\vec{L} \vec{\varphi})_{\mathbf{1}}(\vec{L} \vec{\varphi})_{\mathbf{1}}
$$

The first type leads to the couplings

$$
\begin{aligned}
& \frac{g_{1}}{\Lambda}\left(\nu_{1} \nu_{1} \varphi_{1} \varphi_{1}+\nu_{2} \nu_{2} \varphi_{2} \varphi_{2}+\nu_{3} \nu_{3} \varphi_{3} \varphi_{3}\right) \\
& \quad+2 \frac{g_{2}}{\Lambda}\left(\nu_{2} \nu_{3} \varphi_{2} \varphi_{3}+\nu_{3} \nu_{1} \varphi_{3} \varphi_{1}+\nu_{1} \nu_{2} \varphi_{1} \varphi_{2}\right),
\end{aligned}
$$

where $\Lambda$ is the cutoff and $\nu_{i}$ and $\varphi_{i}$ represent the component $\left(\nu_{L}\right)_{i}$ in $\vec{L}_{i}$ and $\varphi_{i}^{0}$ in $\vec{\varphi}_{i}$, respectively. When the fields $\varphi_{i}^{0}$ have the vacuum expectation values $u_{i}(i=1,2,3)$, the above terms induce the mass matrix term

$$
M_{\nu}^{(a)}=\frac{1}{\Lambda}\left(\begin{array}{ccc}
g_{1} u_{1}^{2} & g_{2} u_{1} u_{2} & g_{2} u_{1} u_{3} \\
g_{2} u_{1} u_{2} & g_{1} u_{2}^{2} & g_{2} u_{2} u_{3} \\
g_{2} u_{1} u_{3} & g_{2} u_{2} u_{3} & g_{1} u_{3}^{2}
\end{array}\right)
$$


for light neutrinos. In the second type, the $\Gamma$-invariant combinations of two singlets $(\vec{L} \vec{\varphi})_{1}$ are $\mathbf{1}_{(k, l)} \times \mathbf{1}_{(-k,-l)}$ with $k, l=0, \pm 1$. Thus the second type operators yields the couplings

$$
\begin{aligned}
& \frac{f_{1}}{\Lambda}\left(\nu_{1} \varphi_{1}+\nu_{2} \varphi_{2}+\nu_{3} \varphi_{3}\right)^{2} \\
& +2 \frac{f_{2}}{\Lambda}\left(\nu_{1} \varphi_{1}+\omega^{2} \nu_{2} \varphi_{2}+\omega \nu_{3} \varphi_{3}\right)\left(\nu_{1} \varphi_{1}+\omega \nu_{2} \varphi_{2}+\omega^{2} \nu_{3} \varphi_{3}\right) \\
& +2 \frac{f_{3}}{\Lambda}\left(\nu_{1} \varphi_{3}+\nu_{2} \varphi_{1}+\nu_{3} \varphi_{2}\right)\left(\nu_{1} \varphi_{2}+\nu_{2} \varphi_{3}+\nu_{3} \varphi_{1}\right) \\
& +2 \frac{f_{4}}{\Lambda}\left(\nu_{1} \varphi_{3}+\omega^{2} \nu_{2} \varphi_{1}+\omega \nu_{3} \varphi_{2}\right)\left(\nu_{1} \varphi_{2}+\omega \nu_{2} \varphi_{3}+\omega^{2} \nu_{3} \varphi_{1}\right) \\
& +2 \frac{f_{5}}{\Lambda}\left(\nu_{1} \varphi_{3}+\omega \nu_{2} \varphi_{1}+\omega^{2} \nu_{3} \varphi_{2}\right)\left(\nu_{1} \varphi_{2}+\omega^{2} \nu_{2} \varphi_{3}+\omega \nu_{3} \varphi_{1}\right) .
\end{aligned}
$$

Taking $f_{3}=f_{4}=f_{5}$, we have another mass matrix term

$$
\begin{aligned}
& M_{\nu}^{(b)}=\frac{1}{\Lambda}\left(\begin{array}{ccc}
\left(f_{1}+2 f_{2}\right) u_{1}^{2} & \left(f_{1}-f_{2}\right) u_{1} u_{2} & \left(f_{1}-f_{2}\right) u_{1} u_{3} \\
\left(f_{1}-f_{2}\right) u_{1} u_{2} & \left(f_{1}+2 f_{2}\right) u_{2}{ }^{2} & \left(f_{1}-f_{2}\right) u_{2} u_{3} \\
\left(f_{1}-f_{2}\right) u_{1} u_{3} & \left(f_{1}-f_{2}\right) u_{2} u_{3} & \left(f_{1}+2 f_{2}\right) u_{3}{ }^{2}
\end{array}\right) \\
& +\frac{1}{\Lambda}\left(\begin{array}{ccc}
6 f_{3} u_{2} u_{3} & 0 & 0 \\
0 & 6 f_{3} u_{3} u_{1} & 0 \\
0 & 0 & 6 f_{3} u_{1} u_{2}
\end{array}\right)
\end{aligned}
$$

We now assume a particular vacuum alignment $u_{1}=0$ and $u_{2}=u_{3}$. In this case the light neutrino mass matrix becomes

$$
\begin{aligned}
M_{\nu} & =M_{\nu}^{(a)}+M_{\nu}^{(b)} \\
& =\frac{u_{2}^{2}}{\Lambda}\left(\begin{array}{ccc}
6 f_{3} & 0 & 0 \\
0 & \left(g_{1}+f_{1}+2 f_{2}\right) & \left(g_{2}+f_{1}-f_{2}\right) \\
0 & \left(g_{2}+f_{1}-f_{2}\right) & \left(g_{1}+f_{1}+2 f_{2}\right)
\end{array}\right) .
\end{aligned}
$$

This mass matrix is diagonalized through the transformation $U_{\nu}^{T} M_{\nu} U_{\nu}$. The unitary 
matrix $U_{\nu}$ is of the form

$$
U_{\nu}=\left(\begin{array}{ccc}
0 & 1 & 0 \\
\frac{1}{\sqrt{2}} & 0 & \frac{-i}{\sqrt{2}} \\
\frac{1}{\sqrt{2}} & 0 & \frac{i}{\sqrt{2}}
\end{array}\right)
$$

The lepton mixing matrix is given by $U_{l \nu}=U_{l}^{\dagger} U_{\nu}$. Referring to Eqs. (20) and (27), we find that the lepton mixing is tri-bimaximal, i.e.,

$$
U_{l \nu}=\left(\begin{array}{ccc}
\sqrt{\frac{2}{3}} & \frac{1}{\sqrt{3}} & 0 \\
-\frac{1}{\sqrt{6}} & \frac{1}{\sqrt{3}} & -\frac{1}{\sqrt{2}} \\
-\frac{1}{\sqrt{6}} & \frac{1}{\sqrt{3}} & \frac{1}{\sqrt{2}}
\end{array}\right)
$$

\section{Summary}

In this paper we have studied the effective theory with the semidirect product gauge group $G=S U(3)_{\mathrm{L}} \rtimes\left(\mathbb{Z}_{3} \times \mathbb{Z}_{3}\right)$. In the semidirect product gauge group we need to introduce the projective homomorphism of $\mathbb{Z}_{3} \times \mathbb{Z}_{3}$ into $S U(3)_{\mathrm{L}}$. In spite of Abelian nature of $\mathbb{Z}_{3} \times \mathbb{Z}_{3}$, there appear flavor triplets. This result is general features of the semidirect product gauge group. We applied this result to the issue of the lepton mixing and gave a toy model. It was shown that under a particular vacuum alignment the tri-bimaximal mixing matrix is reproduced.

It is feasible that the semidirect product gauge group $G=G_{c} \rtimes \Gamma$ is traced back to a unified gauge group $\tilde{G}$. Namely, there is a possibility that the symmetry breaking of $\tilde{G}$ down to $G=G_{c} \rtimes \Gamma$ takes place via the Higgs mechanism. A simple example illustrative of such breaking structure is the symmetry breaking $S O(3) \rightarrow S O(2) \rtimes \mathbb{Z}_{2}$ which has been discussed by Preskill and Krauss. [7] The theories with the semidirect product gauge group have more attractive and rich structure compared to those with the direct product gauge group. It is expected that the study of theories with the semidirect product gauge group sheds new light on the development of gauge theories. 


\section{Acknowledgements}

One of the authors (M. M.) is grateful to Jyun'ichi Iwamoto and Shoushirou Sugio for discussions at the early stage of this research.

\section{References}

[1] P. H. Harrison, D. H. Perkins and W. G. Scott, Phys. Lett. B530 (2002), 167, arXiv:hep-ph/0202074.

[2] For example, see the following review papers and references therein.

C. H. Albright, Talk given at 13th International Workshop on Neutrino Telescopes, 2009, arXiv:0905.0146[hep-ph]],

E. Ma, arXiv:0905.0221[hep-ph],

G. Altarelli, Talk given at 13th International Workshop on Neutrino Telescopes, 2009, arXiv:0905.2350[hep-ph]],

S. F. King, Plenary talk given at SUSY'09, arXiv:0909.2969[hep-ph]].

[3] T. Banks, Physicalia Magazine 12 (1990), 19.

[4] C. Hattori, M. Matsunaga, T. Matsuoka and K. Nakanishi, in preparation.

[5] G. Karpilovsky, Projective Representations of Finite Groups, Pure and Applied Math., Marcel Dekker, Inc., 1985, §3.7.

[6] S. Mosiri and E. Peinado, arXiv:0910.4389[hep-ph].

[7] J. Preskill and L. M. Krauss, Nucl. Phys. B341 (1990), 50. 